Musées, Patrimoine et Culture scientifiques et techniques

$132 \mid 2010$

novembre - décembre 2010

\title{
Le texte dans l'exposition
}

Text' in an exhibition context

\section{Marie-Sylvie Poli}

URL : http://journals.openedition.org/ocim/376

DOI : 10.4000/ocim.376

ISSN : 2108-646X

Éditeur

OCIM

Édition imprimée

Date de publication : 1 novembre 2010

Pagination : 5-7

ISSN : 0994-1908

Référence électronique

Marie-Sylvie Poli, « Le texte dans l'exposition », La Lettre de I'OCIM [En ligne], 132 | 2010, mis en ligne le 01 novembre 2012, consulté le 19 avril 2019. URL : http://journals.openedition.org/ocim/376 ; DOI :

10.4000/ocim.376

Ce document a été généré automatiquement le 19 avril 2019.

Tous droits réservés 


\title{
Le texte dans l'exposition
}

\author{
Text' in an exhibition context
}

\author{
Marie-Sylvie Poli
}

1 Les auteurs des articles qui composent ce numéro thématique consacré au texte dans l'exposition sont intervenus lors d'un stage de formation de l'OCIM Le texte dans l'exposition (adultes et enfants) organisé à Grenoble du 16 au 18 septembre 2008 au musée de la Résistance et de la Déportation de l'Isère, au musée Dauphinois et à la Casemate/ CCSTI de Grenoble. Ce n'était pas la première session de ce type centrée sur la question du texte écrit et on peut sans prendre de risque penser que l'OCIM répondra encore à l'avenir aux demandes récurrentes des professionnels - concepteurs d'exposition et médiateurs, surtout - d'organiser des temps de réflexion et de formation consacrés à la problématique du texte dans l'exposition. Chaque session de stage développe d'ailleurs des approches et des modalités de travail différentes, tant la thématique du texte au musée s'avère toujours ample et complexe, aussi bien théorique que concrète.

2 Lors de cette session, des chercheurs (sémiologues et sociologues) et des professionnels de la médiation (responsables des publics, concepteurs d'exposition, médiateurs) sont intervenus sur la question du texte comme support langagier de médiation du sens dans l'exposition, en partant systématiquement de recherches ou d'expériences de terrain sur lesquelles ils avaient récemment travaillé.

3 Marie-Sylvie Poli (muséologue et responsable scientifique de la formation) aborde la question des mécaniques linguistiques de production du texte dans l'exposition : la forme linguistique n'est pas un habillage du sens, elle est à la fois la condition et le lieu de production du sens. Cette approche qui pense toujours le texte intégré aux autres registres de signes de l'exposition, permet d'insister sur les contraintes de ce genre d'écrit qui, bien que connues, ne cessent d'évoluer et de se transformer au gré de la créativité des auteurs. Elle rappelle que du point de vue de la communication, considérer le texte comme un système autonome clos sur lui-même dans l'exposition s'avère n'avoir aucun sens. Le texte joue aussi dans la cour de la scénographie, des objets, des espaces et des ambiances. Mais elle souligne aussi que négliger la force d'impact du style et des 
formes du texte (écrit ou dit) n'a pas de sens non plus. Écrire pour l'exposition relève du même défi qu'écrire en règle générale : il s'agit d'une aventure centrée sur la dimension communicationnelle et poétique de la langue.

4 Pascale Ancel (sociologue) traite la question plus générale de la réception du texte par les publics adultes. En s'appuyant sur trois études de publics menées dans trois expositions de la région grenobloise (musée de la Révolution de Vizille, musée de Grenoble et CCSTI) elle montre que les visiteurs ne limitent pas leur représentation du texte dans l'exposition à une dimension strictement informative ou pédagogique. Derrière les mots et les phrases, les visiteurs perçoivent les intentions culturelles des auteurs des dispositifs d'écrit à l'égard de leur public. Question d'attente vis-à-vis du musée ? Certes, mais pas seulement. Ces études démontrent aussi que les visiteurs interprètent les textes de médiation comme un type de discours spécifique qui permet aux institutions muséales de défendre leur engagement scientifique et culturel.

5 Á la Casemate/CCSTI de Grenoble, Armelle Chaléon (animatrice jeune public), Catherine Demarcq (responsable du service animation) et Ludovic Maggioni (chargé de projet d'exposition) font part de leur tout récent travail d'équipe pour créer et réaliser divers supports textuels destinés aux jeunes publics venus au CCSTI pour visiter l'exposition SOS Pôles, le changement climatique en question en contexte scolaire et en contexte familial. Ils expliquent pourquoi, comment et avec quels outils ils ont décidé de multiplier les écrits distribués aux jeunes visiteurs lors des visites guidées, pour animer des ateliers science et créer des jeux (pour trois tranches d'âge). L'expérience méritait d'autant plus d'être relatée que ces multiples dispositifs de textes conçus par l'équipe de la Casemate ont été créés pour adapter au format exposition grand public, un dossier « Expo Science Actualités " proposé par la Cité des Sciences et de l'Industrie. C'est donc autant la question de la réécriture des outils d'animation dans une exposition de sciences et société itinérante qui est abordée, que celle de l'écriture.

6 Franck Philippeaux (responsable des publics et du site Internet au musée Dauphinois) rappelle combien pour le musée Dauphinois le texte est un objet et un terrain d'innovation permanent, que les concepteurs d'exposition remettent en question à chaque projet, en fonction du sujet de l'exposition et des publics spécifiquement visés, comme le public enfant par exemple. Pour approfondir ce sujet précis, il présente une évaluation de remédiation en cours : chercher à améliorer un dispositif de médiation écrit destiné aux enfants de huit à douze ans (textes, visuels et parcours jeux) dans Gens de l'alpe l'exposition phare de ce musée de société, en tenant compte à la fois des études précédemment menées au musée Dauphinois sur l'écrit en général et de la volonté d'innover en matière d'aide à la visite en autonomie pour les enfants. Ce témoignage permet de mettre l'accent sur l'intérêt et la difficulté pour le service des publics, de mener régulièrement des évaluations des outils de médiation destinés aux enfants, par des enquêtes qualitatives réalisées auprès des jeunes visiteurs et des adultes qui les accompagnent.

7 Françoise Rigat (linguiste et muséologue italienne) montre combien la traduction des textes pour des visiteurs étrangers ne peut en aucun cas se limiter à une banale opération de traduction automatique du français en anglais ou en italien. À partir d'exemples précis repérés dans des expositions dont elle a travaillé les thèmes et les outils de médiation plurilingues, elle prouve que la traduction mérite d'être envisagée et traitée comme une médiation à part entière, par tous les acteurs de l'exposition, et à toutes les étapes du projet. 
8 Valérie Chauvey (médiatrice spécialiste des publics à handicap visuel) présente des notions théoriques fondamentales (psychologie, sémiologie) et une analyse critique de dispositifs - rarement évalués (braille, audio, livrets, Internet) - proposés à des visiteurs de musée non-voyants.

En marge des contributions écrites présentées dans ce numéro de la Lettre de l'OCIM, deux intervenants ont enrichi les travaux du stage. Voici la synthèse de leur communication orale.

10 Jean-Claude Duclos est intervenu en tant que conservateur en chef du Patrimoine et directeur du musée de la Résistance et de la Déportation de l'Isère (MRDI). Á ce titre il a tenu à insister sur la dimension systémique des obligations - ou contraintes - auxquelles doit toujours faire face le commissaire d'exposition, auteur des textes dans un musée comme le MRDI. Tout d'abord respecter la rigueur scientifique des contenus et des informations exposés sans déroger aux engagements muséaux de diffusion de ces savoirs vers un large public. Ce qui implique une écriture qui dialogue avec les communautés (résistants, militants, citoyens) ou les spécialistes (historiens, anthropologues, artistes) avec qui tout projet d'exposition est travaillé de manière participative, au moins six mois avant toute inauguration (parfois bien plus longtemps). Ensuite inscrire par le texte l'exposition dans une temporalité qui est autant scientifique (les savoirs et les conceptions à un moment donné) qu'institutionnelle (les soutiens des tutelles comme le Conseil général de l'Isère, la Ville de Grenoble, l'État...). Enfin tenir compte de tous les publics auxquels l'exposition est destinée en prenant garde à préserver un juste équilibre entre les écrits du musée, le discours scientifique (histoire) et la parole des témoins (mémoire) invités à s'exprimer dans les expositions de ce musée qui revendique sa place de musée d'histoire autant que de musée de société. Il a également insisté sur l'intérêt des études de réception dans un musée comme le MRDI, des recherches de terrain qui permettent aux concepteurs de mieux connaître les réactions des visiteurs (adultes et jeunes publics) aux textes écrits qui leur sont proposés, mais également à tous les dispositifs (audio et vidéo, aides à la visite) qui produisent du discours sur des objets, des concepts, des archives, des événements, des personnes.

11 Delphine Miège (responsable médiation et docteur en Communication) a pris le prétexte de l'exposition d'art contemporain pour aborder de manière très approfondie la question complexe du discours rapporté dans l'exposition. Dans quelle mesure le public (à qui s'adressent les textes qui citent plus ou moins explicitement un artiste ou un critique d'art) trouve-t-il intérêt face à certains jeux de style parfois très raffinés ? Faut-il systématiquement donner les références des auteurs que l'on fait parler lorsqu'on écrit du texte d'aide à la visite ? Ou encore, est-il parfaitement légitime pour un commissaire d'exposition de s'approprier des propos pour lesquels il crée une nouvelle pertinence en les insérant dans un discours d'exposition approprié à une thématique, à une temporalité ? Á partir d'exemples précis et variés, elle a démontré que bien souvent le discours rapporté se fond dans le texte d'exposition, au point qu'il est difficile aux non experts de retrouver la trace des textes sources. 
INDEX

Mots-clés : exposition, texte

AUTEUR

MARIE-SYLVIE POLI

Professeur à l'université Pierre Mendès France, Grenoble 